\title{
Omalizumab for refractory chronic spontaneous urticaria during concurrent immunomodulatory therapy for multiple sclerosis
}

\author{
${ }^{1}$ Allergy Department, "Sotiria” General Hospital, Athens, Greece \\ ${ }^{2} 3^{\text {rd }}$ Department of Medicine, "Sotiria" General Hospital, Medical School, University of Athens, Athens, Greece
}

\author{
KEY WORDS \\ angioedema; antihistamines; \\ chronic spontaneous urticaria; \\ omalizumab; multiple sclerosis. \\ Corresponding author \\ Dimitra Grapsa \\ $3^{\text {rd }}$ Department of Medicine, "Sotiria" \\ General Hospital \\ University of Athens, School of Medicine, \\ Athens, Greece \\ Mesogion 152, 11527 Athens, Greece \\ Phone: +30 2107751063 \\ Fax: +30 2107751063 \\ E-mail: dimgrap@yahoo.gr
}

Doi

10.23822/EurAnnACI.1764-1489.20

\begin{abstract}
Summary
Data derived from previous clinical trials and real-life studies have shown that omalizumab may represent an effective third-line treatment option for patients with chronic spontaneous urticaria (CSU) refractory to standard antihistamine treatment. Nevertheless, the safety and efficacy of omalizumab treatment for CSU, when administered concurrently with other immunomodulatory agents remains largely unknown. We herein present the case of a female patient with relapsing-remitting multiple sclerosis (RRMS), under treatment with interferon beta-1a, azathioprine and gabapentin, who was successfully treated with omalizumab for refractory CSU. To the best of our knowledge, this is the first reported case attesting to the safety and efficacy of omalizumab for CSU when administered concurrently with other immunomodulatory agents.
\end{abstract}

Omalizumab is a humanized anti-IgE monoclonal antibody currently approved by the Food and Drug Administration (FDA) for the treatment of moderate to severe allergic asthma and chronic spontaneous urticaria (CSU) not responding to standard antihistamine treatment. Off-label uses of omalizumab include an expanding list of various allergic and autoimmune conditions, while its exact mechanism of action, as well as its potential synergies or interactions with other drugs, remain relatively unknown (1). To the best of our knowledge, there are no previous reports supporting the efficacy and safety of omalizumab when administered concurrently with other immunomodulatory agents. We herein present the clinical presentation, course and outcome of a patient with relapsing-remitting multiple sclerosis (RRMS), under immunomodulatory therapy, who was successfully treated with omalizumab for refractory CSU.
A 53-year old female, with a 7-year history of RRMS - treated with interferon beta-1a, azathioprine and gabapentin - presented to the Allergy Department of Sotiria Athens General Hospital, with widespread urticaria and angioedema lasting for 12 weeks. Her remaining medical history was remarkable for essential hypertension, treated with amlodipine / valsartan. Two weeks after appearance of urticaria, all drugs for MS and hypertension were discontinued, with no resolution of symptoms, and reinstated approximately four weeks later, again with no symptomatic change. Following administration of first-line antihistamine monotherapy (levocetirizine, $10 \mathrm{mg}$ once daily) and repeated courses of corticosteroids (oral methylprednisolone, 64 $\mathrm{mg} /$ day and 6 intramuscular injections of hydrocortisone, 250 $\mathrm{mg}$ /injection), no significant symptomatic improvement was noted and the patient was referred by her primary care phy- 
sician to an allergy clinic for further diagnostic evaluation and therapeutic management.

At the time of the patient's presentation to our department a comprehensive investigation for potential underlying causes of chronic urticaria was initiated. More specifically, a detailed clinical history, physical examination, chest X-ray and thorough laboratory testing, including complete blood count with differential, complete biochemical profile, erythrocyte sedimentation rate, C-reactive protein and thyroid-stimulating hormone levels (TSH), failed to reveal any clinically relevant findings; serological tests for hepatitis B surface antigen (HBsAg), human immunodeficiency virus (HIV), thyroid autoantibodies (anti-thyroid peroxidase and anti-thyroglobulin antibodies), antinuclear antibodies (ANA), anti-double stranded DNA (anti-dsDNA) antibodies and rheumatoid factor, were all negative. Serum immunoglobulins (IgG, IgA, IgM and IgE), complement C3 and $\mathrm{C} 4$ and rheumatoid factor levels were all within normal limits. Autologous serum skin test (ASST) was also performed, with negative results. Treatment was modified with switch from levocetirizin to cetirizine ( $20 \mathrm{mg} \mathrm{x} 4$ daily), and subsequent addition of montelukast (10 mg once daily) and ranitidine (150 mg twice daily), with no significant improvement. An additional 9-day course of methylprednisolone (total dose of $188 \mathrm{mg}$ ) also failed to significantly improve symptoms. The patient was thereafter placed on omalizumab treatment $(300 \mathrm{mg}$ by subcutaneous injection every 4 weeks), with complete remission of symptoms within 2 days following administration of the first dose. At the time of her latest follow up visit, six months after initiation of omalizumab treatment, no recurrence of urticaria/angioedema, significant toxicity or alteration of her neurological status, as further confirmed by her attending neurologist, were noted.

CSU, otherwise known as chronic idiopathic urticaria (CIU), is a condition characterized by spontaneous appearance of itchy wheals (hives), angioedema, or both, that recur for a period longer than 6 weeks and have no identifiable external trigger $(2,3)$. Data derived from phase III clinical trials have demonstrated that omalizumab may represent a safe and effective third-line treatment option in CSU (4-6). Nevertheless, the short- and long-term safety and efficacy of this drug in CSU patients with comorbidities treated with other biologic response modifiers remains essentially unexplored.

Multiple sclerosis (MS) is a chronic inflammatory demyelinating disease of the central nervous disease, with RRMS as its most common form. Treatment for RRMS is primarily aimed at reducing the frequency of relapses by suppressing or modulating the immune system response (7). Various immunomodulatory and immunosuppressive agents are now available for RRMS, including, among others, injectable drugs with well-established safety and efficacy, such as interferon beta-1a, azathioprine and glatiramer acetate, as well as new therapies such as fingolimod, the first oral drug approved for the treatment of MS, or monoclonal antibodies, such as natalizumab, carrying the potential of higher efficacy but, also, an increased risk of serious complications (7). Our reported case attests to the short-term efficacy and safety of omalizumab treatment for CSU when administered concurrently with interferon beta-1a and/or azathioprine. Additional post-marketing safety data regarding concomitant use of omalizumab with other immunomodulatory agents are warranted.

\section{Summary statement}

The safety and efficacy of omalizumab treatment for refractory CSU in patients receiving other immumodulatory agents has not been previously evaluated. We herein report the case of a patient with refractory CSU successfully treated with omalizumab, while receiving concurrent immunomodulatory treatment for RRMS.

\section{References}

1. El-Qutob C. Off-label uses of omalizumab. Clin Rev Allergy Immunol. 2016;50:84-96.

2. Zuberbier T, Aberer W, Asero R et al. The EAACI/GA(2)LEN/ $\mathrm{EDF} / \mathrm{WAO}$ guideline for the definition, classification, diagnosis and management of urticaria: the 2013 revision and update. Allergy. 2014;69:868-87.

3. Bernstein JA, Lang DM, Khan DA et al. The diagnosis and management of acute and chronic urticaria: 2014 update. Journal of Allergy and Clinical Immunology. 2014;133:1270-7.

4. Kaplan A, Ledford D, Ashby M et al. Omalizumab in patients with symptomatic chronic idiopathic/spontaneous urticaria despite standard combination therapy. Journal of Allergy and Clinical Immunology. 2013;132:101-9.

5. Maurer M, Rosen K, Hsieh $\mathrm{HJ}$ et al. Omalizumab for the treatment of chronic idiopathic or spontaneous urticaria. New England Journal of Medicine. 2013;368:924-35.

6. Saini SS, Bindslev-Jensen C, Maurer M et al. Efficacy and safety of omalizumab in patients with chronic idiopathic/ spontaneous urticaria who remain symptomatic on $\mathrm{H} 1$ antihistamines: a randomized, placebo-controlled study. Journal of Investigative Dermatology. 2015;135:925.

7. Cross AH, Naismith RT. Established and novel disease-modifying treatments in multiple sclerosis. Journal of Internal Medicine. 2014;275:350-63. 